\title{
Misplacement of Tracheostomy Tube in the Right Main Bronchus: a Rare Complication
}

\author{
Armin Amirian, ${ }^{1}$ Reza Shahriarirad $\mathbb{D}^{1,2}$ Bizhan Ziaian, ${ }^{1,3}$ Parviz Mardani,, \\ and Amirhossein Erfani (D) 1,2 \\ ${ }^{1}$ Thoracic and Vascular Surgery Research Center, Shiraz University of Medical Science, Shiraz, Iran \\ ${ }^{2}$ Student Research Committee, Shiraz University of Medical Sciences, Shiraz, Iran \\ ${ }^{3}$ Department of Surgery, Shiraz University of Medical Sciences, Shiraz, Iran
}

Correspondence should be addressed to Reza Shahriarirad; r.shahriari1995@gmail.com

Received 10 December 2019; Revised 4 January 2020; Accepted 5 February 2020; Published 14 February 2020

Academic Editor: Mario Ganau

Copyright ( 2020 Armin Amirian et al. This is an open access article distributed under the Creative Commons Attribution License, which permits unrestricted use, distribution, and reproduction in any medium, provided the original work is properly cited.

\begin{abstract}
A 38-year-old woman known case of metastatic squamous cell carcinoma of the cervical esophagus due to increasing dyspnea and stridor attributed to the pressure effect of the primary mass was scheduled for tracheostomy, which ended up in the right main bronchus. This rare complication occurred using a tracheostomy tube number 7.5 via a vertical tracheotomy over 4 th and 5 th tracheal rings. The misplacement was confirmed by chest X-ray and fiberoptic bronchoscopy, and the tracheostomy tube was successfully repositioned in a nonoperative approach.
\end{abstract}

\section{Introduction}

Unrecognized placement of a tracheostomy tube into the main bronchus is potentially catastrophic. A recent report of a near disaster following tracheostomy insertion in a short-stature lung cancer patient in which the tracheal tube inadvertently entered the right main bronchus, prompted this account, which illustrates, in a spontaneously breathing patient, the necessity of post tracheostomy insertion imaging for evaluation of proper tracheostomy tube location in cases of challenging anatomy or otherwise difficult tracheostomy tube placement.

\section{Case Presentation}

A 38-year-old woman known case of metastatic squamous cell carcinoma of the cervical esophagus was scheduled for tracheostomy due to increasing dyspnea and stridor attributed to the pressure effect of the primary mass. The patient reported dysphagia for 3 months prior to seeking medical advice and upper GI endoscopy was done 2 weeks before admission, which reported near-complete obstruction of the esophageal lumen at the cricopharyngeus level (UES) from which the scope could not pass. So multiple biopsies were done which reported poorly differentiated squamous cell carcinoma. When the patient came to our center, she had severe stridor and critical airway; thus, we had no time for any further workup and immediate transport to OR and intubation by an anesthesiologist was done. Orotracheal intubation was done with the guide of a fiberoptic bronchoscope, and an uncuffed endotracheal tube (ETT) number 5 was passed beyond the stenotic segment of the upper trachea. Unfortunately, only standard SUPA tracheostomy tubes with a fixed length, but variable diameters, are available in our OR stoke, due to Shiley tubes are not easily available in our country. A $3 \mathrm{~cm}$ transverse incision was made $1 \mathrm{~cm}$ above the sternal notch, and a cuffed tracheostomy tube number 7.5 was inserted via a vertical tracheotomy over the 4 th and 5th rings of the trachea, guided by manual palpation of tracheal cartilages which were hard and fixed above this level. Proper position was confirmed with end-tidal $\mathrm{CO}_{2}$, and the patient was transferred to the recovery unit with high-flow oxygen applied via T-piece connected to the tracheostomy tube. Flexible bronchoscopy was not done due to no findings in favour of misplacements, such as difficult ventilation. Upon arrival in the recovery unit and after disconnecting from T-piece, the patient developed dyspnea and $\mathrm{O}_{2}$ saturation 


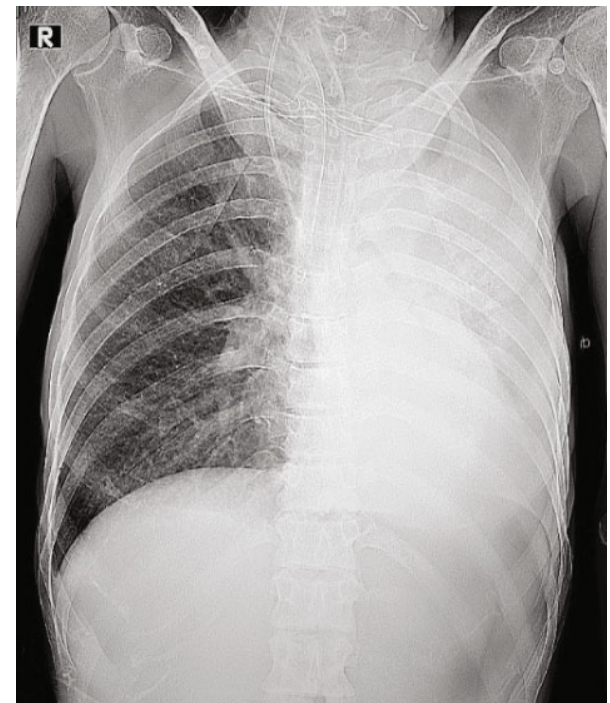

FIgURe 1: Post tracheostomy insertion portable CXR.
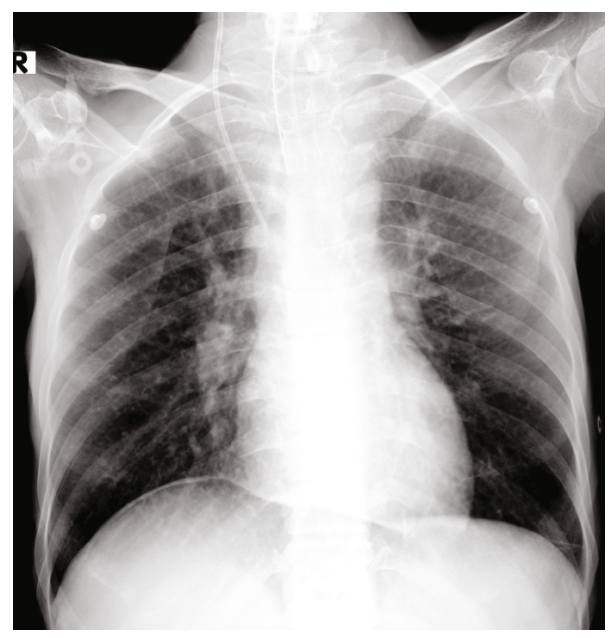

Figure 2: Chest X-ray after adjusting tracheostomy tube.

dropped to $80 \%$. Physical exam showed tachypnea and poor expansion of the left lung with absent breathing sounds. An urgent portable chest X-ray (CXR) was done which revealed left hemithorax white out and tip of ETT placed in the right mainstem bronchus (Figure 1). The patient was immediately returned to the operating room, and fiberoptic bronchoscopy was done from the tracheostomy tube which confirmed misplacement of the tube in the right main bronchus. The tracheostomy tube was withdrawn above the carina under the guide of a bronchoscope, and two gauzes were used to fix it in the new position. General condition improved as documented by bilateral lung expansion and a rise in $\mathrm{O}_{2}$ saturation. Repeat CXR in recovery confirmed the appropriate position of the tracheostomy tube and left lung expansion (Figure 2).

\section{Discussion}

Patients with obstruction in the pharynx or the laryngeal area of the throat usually require a bypass route to supply the necessary air or mixture of gases, in which tracheostomy tubes are in use. For implantation of the tube, an incision is made below the obstructed area in the neck of the patent in which the distal end of an outer cannula is cautiously inserted into the trachea, and in order to provide air passage into the trachea, the proximal end of the outer cannula remains outside the trachea in communication with ambient air. The use of tracheostomy to provide long-term airway management in patients, although common, has been related to complication rates that differ based on the type of procedure implemented and clinical settings [1,2]. Additional experience with the procedure and careful patient selection may decrease complication rates to an acceptable level [1]. Some studies have suggested that most of the injuries occur in short-stature female patients [3].

Cornelius et al. reported a case of misplacement of the ETT into the right bronchus due to a short neck length of the patient. Greater appreciation and vigilance of the possible risk of bronchial intubation due to this anatomical feature is warranted [4].

Medical imaging accounts for a large element of health care spending. Usually, imaging adds to quality medical care. Nevertheless, imaging can cause considerable increases in medical costs without improving diagnostic yields when used inappropriately [2].

Although the use of imaging after tracheostomy tube insertion has been a controversial issue, a review article on routine daily chest radiography in patients with tracheostomy tubes showed that only $2.3 \%$ of radiographs revealed new complications which resulted in a $0.7 \%$ management change [2]. Therefore, accurate patent selection based on the patients' clinical status is essential due to routine chest radiography after tracheostomy does not appear to change patient management regularly enough to warrant the procedure expenditures.

Post intubation CXR seems to be limited to patients undergoing technically problematic and complicated procedures. Based on the incidence of malpositioned tubes, post endotracheal intubation chest radiography is supported by numerous studies [5-9]. The recommendation for routine CXR after tracheostomy is indeed controversial in uncomplicated tracheostomy placement; however, when there is a difficulty due to anatomic variance, tumor, or revisions, then CXR is certainly beneficial to confirm appropriate tube placement. This recommendation also is based on the incapability of physicians to foresee complications based on clinical features [5-7]. However, the procedure of post tube thoracostomy chest radiography is reinforced due to the fact that complications of inappropriate intubations are highly remarkable and CXR remains a necessary step to minimize the misplacement of the tube [10].

\section{Conclusion}

In conclusion, physicians should have in mind the complications of tracheostomy and the necessity of CXR after tracheostomy tube insertion due to the possibility of bronchial misplacement, which has also been widely reported in the case of ETT, which is more likely in short-stature patients 
and tracheostomy insertions that are performed below the normal site due to presence of tumor.

\section{Consent}

Consent was obtained from the patient regarding the publication of this case report.

\section{Conflicts of Interest}

The authors declare that they have no competing interests.

\section{Authors' Contributions}

AA, MP, and ZB collected the data. RS and AE drafted the manuscript. AA revised and proofread the manuscript. All authors read and approved the final version of the manuscript.

\section{Acknowledgments}

The technical assistant of staff in Namazi hospital is acknowledged.

\section{References}

[1] S. Fernandez-Bussy, B. Mahajan, E. Folch, I. Caviedes, J. Guerrero, and A. Majid, "Tracheostomy tube placement: early and late complications," Journal of Bronchology \& Interventional Pulmonology, vol. 22, no. 4, pp. 357-364, 2015.

[2] J. Cruz, M. Ferra, A. Kasarabada, J. Gasperino, and B. Zigmund, "Evaluation of the clinical utility of routine daily chest radiography in intensive care unit patients with tracheostomy tubes: a retrospective review," Journal of Intensive Care Medicine, vol. 31, no. 5, pp. 333-337, 2016.

[3] G. Massard, J. Wihlm, N. Roeslin et al., "Iatrogenic tracheobronchial lesions during intubation. 5 cases and review of the literature," Journal de Chirurgie, vol. 129, no. 6-7, pp. 297302, 1992.

[4] B. Cornelius and T. Sakai, "Inadvertent endobronchial intubation in a patient with a short neck length," Anesthesia Progress, vol. 62, no. 2, pp. 66-70, 2015.

[5] W. Brunel, D. L. Coleman, D. E. Schwartz, E. Peper, and N. H. Cohen, "Assessment of routine chest roentgenograms and the physical examination to confirm endotracheal tube position," Chest, vol. 96, no. 5, pp. 1043-1045, 1989.

[6] P. Gray, G. Sullivan, P. Ostryzniuk, T. McEwen, M. Rigby, and D. E. Roberts, "Value of postprocedural chest radiographs in the adult intensive care unit," Critical Care Medicine, vol. 20, no. 11, pp. 1513-1518, 1992.

[7] J. E. Heffner and J. Klein, "Chest imaging in critically ill patients: analysis of clinical value," Respiratory Care, vol. 39, no. 1, pp. 51-62, 1994

[8] H. Hossein-Nejad, P. Payandemehr, S. A. Bashiri, and H. H.-N. Nedai, "Chest radiography after endotracheal tube placement: is it necessary or not?" The American Journal of Emergency Medicine, vol. 31, no. 8, pp. 1181-1182, 2013.
[9] W. Geisser, D. Maybauer, H. Wolff, E. Pfenninger, and M. Maybauer, "Radiological validation of tracheal tube insertion depth in out-of-hospital and in-hospital emergency patients," Anaesthesia, vol. 64, no. 9, pp. 973-977, 2009.

[10] M. Tarnoff, M. Moncure, F. Jones, S. Ross, and M. Goodman, "The value of routine posttracheostomy chest radiography," Chest, vol. 113, no. 6, pp. 1647-1649, 1998. 